\title{
Towards Cross-Organizational Innovative Business Process Interoperability Services
}

\author{
Ömer Karacan ${ }^{1}$, Enrico Del Grosso ${ }^{2}$, Cyril $\mathrm{Carrez}^{3}$, and Francesco Taglino ${ }^{4}$ \\ ${ }^{1}$ Siemens AG, Vienna, Austria \\ oemer. karacan@siemens.com \\ 2 TXT e-Solutions, Milano, Italy \\ enrico.delgrosso@txt.it \\ ${ }^{3}$ SINTEF ICT, Oslo, Norway \\ Cyril.Carrezasintef.no \\ ${ }^{4}$ CNR-IASI, Roma, Italy \\ francesco.taglino@iasi.cnr.it
}

\begin{abstract}
This paper presents the vision and initial results of the COIN (FP7-IST216256) European project for the development of open source Collaborative Business Process Interoperability (CBPip) in cross-organisational business collaboration environments following the Software-as-a-Service Utility (SaaS-U) paradigm.
\end{abstract}

Keywords: Interoperability, business, collaborative process, COIN.

\section{Introduction}

COIN (FP7-216256) [1] is an integrated project in the European Commission Seventh Framework Programme. The mission of the COIN project is to study, design, develop and prototype an open, self-adaptive, generic ICT solution where Enterprise Collaboration (EC) and Enterprise Interoperability (EI) services will be an invisible, pervasive and self-adaptive knowledge and business utility at the disposal of the European networked enterprises [13].

In this paper we position the vision and initial results for the development of open source Collaborative Business Process Interoperability (CBPip) services following the Software-as-a-Service Utility (SaaS-U) paradigm. SaaS is a model of software deployment where an application is hosted as a service provided to customers across the Internet, while SaaS-U brings in the picture a new field of interoperability among collaborative enterprises, hence SaaS becomes a utility for them.

The Collaborative Business process (CBP) interoperability is here meant to support cross organizational BP modelling by means of sharing, publishing, and transforming existing business process models.

This paper is structured as follows:

- In section 2 we give a short overview of related work and the context of the CBPip.

- $\quad$ Section 3 describes the prerequisites and concepts for implementing a first set of CBPip services. 
- In section 4 we discuss how the CBPip services fit with the SaaS-U paradigm.

- Section 5 describes the status quo of the ongoing activities and preliminary results.

- $\quad$ Conclusions and future work are presented in section 6.

\section{Related Work}

The mission of COIN is to study, design, develop and prototype an open, selfadaptive, generic ICT integrated solution, starting from notable existing researchïresults in the field of Enterprise Interoperability (specially ATHENA [3], and SHAPE [4]) and Enterprise Collaboration (specially ECOLEAD [2]).

In particular, a COIN business-pervasive open-source service platform will be able to expose, integrate, compose and mash-up in a secure and adaptive way existing and innovative to-be developed Enterprise Interoperability and Enterprise Collaboration services, by applying intelligent maturity models, business rules and self-adaptive decision-support guidelines to guarantee the best combination of the needed services in dependence of the business context, as industrial sector and domain, size of the companies involved, openness and dynamics of collaboration. This way, the Information Technology vision of Software as a Service (SaaS) will find its implementation in the field of interoperability among collaborative enterprises, supporting the various collaborative business forms, from supply chains to business ecosystems, and becoming for them like a utility, a commodity, the so-called Interoperability Service Utility (ISU)[24].

The COIN project will finally develop an original business model based on the SaaS-U paradigm where the open-source COIN service platform will be able to integrate both free-of-charge and chargeable, open and proprietary services depending on the case and business policies.

\section{Collaborative Business Process Interoperability}

The work in CBPip starts with the consolidation of open source results from the previous Enterprise Interoperability research, in particular the ATHENA project (FP6507849) [3]. The ATHENA project was the integrated project focusing on enterprise interoperability in FP6. The project developed an interoperability framework baseline with a set of models, tools, services and methods to solve interoperability issues.

The work in CBPip inherits the results of the ATHENA project as prerequisites and introduces innovative enhancements, particularly business process interoperability according to SaaS-U paradigm.

There are two main CBPip project threads to develop the innovative enhancements. The first thread aims to develop innovative Collaborative Business Process Interoperability (CBPip) web services for business process modeling, semantic annotation and mediation, in order to allow a (semi-) automatic reconciliation between enterprise 
private processes (PP) and their public views, so-called view processes (VP). The ATHENA CBP approach helps us to define a procedure for low-level approach to design the CBP independently and for high-level approach to allow participants to expose to collaboration the view processes of their private processes and to let the CBP to be built interactively. It helps us to conceptualize the top-down Vertical Business Process Interoperability (VBPI) strategy.

The second thread of CBPiP aims to study and experiment process and workflow mining techniques to implement a vertical, bottom-up approach to align the business process interoperability models with the execution traces; the bottom-up Vertical Business Process Interoperability (VBPI) strategy.

\subsection{Top-Down Vertical Business Process Interoperability}

This section outlines the specification of an open source top-down Vertical Business Process Interoperability Services Framework. It takes the ATHENA CBP approach as a starting point and extends it with new novel approaches and EI services, offered as (Semantic) Web services and GUI front-ends (rich clients) according to SaaS and SaaS-U, to support Business Interoperability between collaborating Small Medium Enterprises (SME).

Figure 1 gives a high-level overview of the CBP approach. The different levels on which CBP modeling is performed (business level, technical level, implementation level) are represented on the vertical axis. On the horizontal axis the different model types of the process view concept are shown.

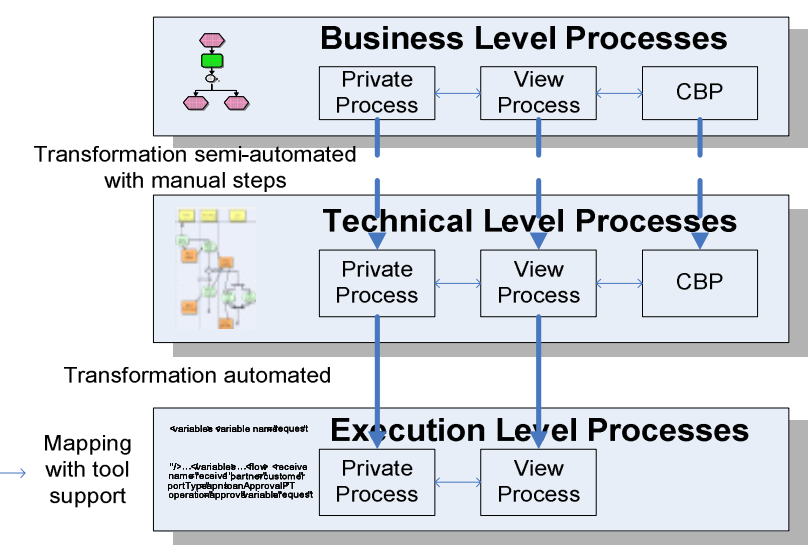

Fig. 1. High-level overview of CBP concepts

The different process levels are:

- Business level processes: This level represents the business views on the collaborative activities, and describes the cross organisational process that defines the interaction among the partners. The CBPs modelled on this level are destined for understanding and governance, and will not in the short term support execution. 
- $\quad$ Technical level processes: This level provides a more detailed view on the CBP representing a more complete control flow of the process. Non-executable tasks are replaced by tasks that can be executed in a system; still the control flow is specified in a platform independent manner. This supports reuse of the process models as the models on this level can then be ported to different process engines on the last level. Also the message exchange between single tasks is modelled on this level.

- Execution level processes: On this level the CBP is modelled in the modelling language of a concrete business process engine. It is extended with platform specific interaction information and data, e.g. the concrete message formats sent or received during $\mathrm{CBP}$ execution or the specification of particular data sources providing data during process execution.

These levels are similar to the different types of models used in OMG model-driven architecture [5], namely computational independent models (CIM), platform independent models (PIM) and platform specific models (PSM). BPMN [6] (possibly extended with some CBP concepts) as a language can be used at the CIM level to express business process models or PIM models to express workflow models that can be (semi)automated and transformed to platform technologies. BPEL [12] is an execution process language that can be used at the PSM level.

At each intersection of a vertical and horizontal axis, we can identify a possible process model to capture tasks and relationships of cross-organizational interactions. Thus it is ensured that all relevant perspectives on CBP models as well as the processes required for the view concept are properly captured and modelled. Transformations between the different modeling levels are necessary. Between the business level and the technical level they can be executed semi-automatically, between the technical level and the execution level they can be automated.

A set of innovative CBPip services provided as (Semantic) Web Services will be the main part of the framework. Ideally, the set of services will need to support the complete CBPip lifecycle. Examples of such services are:

- $\quad$ Set up and manage cross-organizational business process in a shared collaborative environment.

- Modelling and developing Private and Public View processes (as part of the internal preparation for creating CBPip):

- View Process (VP) creation.

- Connect VPs to the shared CBPip.

- Synchronization of CBPip models with external views.

- Modelling and developing shared CBPip: align document exchange in CBPip process parts.

- Execution of the CBPip, i.e., model transformations to executable platforms must be provided.

- $\quad$ Post-execution analysis of modelled CBPip.

- Infrastructure services for storing and executing the CBPip and their corresponding executables.

- Management and monitoring of CBPip. 
These services can be used by either:

- $\quad$ Existing and commercial tools of the collaborating SMEs

- GUI front-ends developed as client-side prototypes as an alternative to existing/commercial tools (where these cannot be used or extended)

In COIN we will aim at leveraging existing technologies and extending them to support additional research issues. For instance, how to accommodate and integrate human-centric processes, e.g., at a PIM-level with BPEL for humans as a target platform. Another extension would focus on how semantic annotations/process models can help us, e.g., can semantic models help us automatically create view processes?

\subsection{Bottom-Up Vertical Business Process Interoperability}

The CBP modelling according to ATHENA is naturally focusing on business processes and collaborative business process modelling on a higher level - on the level CIM of OMG MDA [9], since they are the basic means of negotiation and communication to reach successful business collaboration. They address how to realize a business goal incorporating business information, business organizations, business partners and business resources (both human and machine). It is a logical step to describe CBP through business process modelling. However, in the CBP, the cross-organizational business process interoperability on the CIM, PIM and PSM levels (e.g. BPMN, SoaML [11] and BPEL respectively) is not the focus; the process interoperability is taken as prerequisite, if not, it is assumed as existing. The CBPip concept handles the business process interoperability conceptually through the introduction of the concept pairs "private process / view process". The view process is a derivation of a specific private process hiding the company's critical information from unauthorized partners: it will represent only the required information for external operations, while hiding internal aspects of the (private) process. A view process is always referenced to its private process, which assures business process interoperability. Figure 2 illustrates the difference between a view process and a private process, and how they are used in the COIN environment. Each company $(A$ and $B$ ) build their own private processes, shown at the bottom of the figure. Using the COIN services (see section 5), each company will specify which parts of their processes they wish to publish, and then transform each process into a View Process, which will then be published in the COIN process repository. As shown on the figure, this View Process contains less operations and sub-processes than the Private Process. View Processes are then composed in the CBPip (upper part of Figure 2).

Additionally, the CBPip provides a modelling technique for studying and illustrating (modelling) business process interoperability challenges (problems and possible solutions), generally, on different levels of abstraction: the CBPip Modeling (CBPipM). The CBPipM offers a unified "look \& feel" in all abstraction levels. This will drive us to define potential interoperability services and service utilities in respect to configuration, customization, testability, simulation (e.g. annotating ontologies, generating interoperability rules and mapping functions) and interoperability reverse engineering alias backward transformation (bottom-up strategy). 


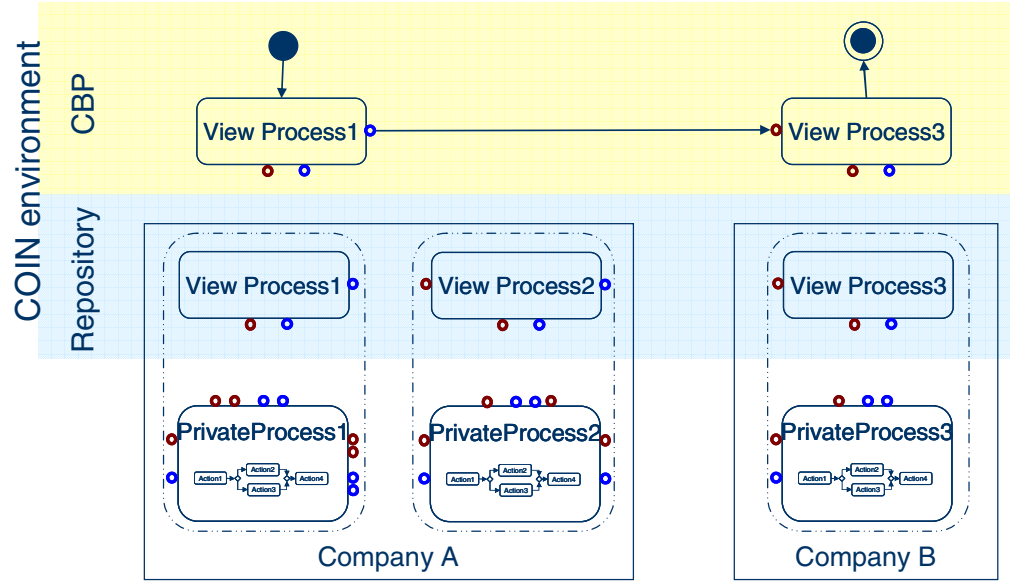

Fig. 2. Private Process and View Process in the COIN environment

Furthermore, CBPipM leverages the artefacts that are undergone a semantic compatibility procedure, where semantic annotation and semantic mediation services are performed.

The concept of CBPipM provides modelling means, where business process interoperability can be defined and modelled from different views and aspects, particularly in the PIM and PSM levels. The CBPipM enables behavioural examination of the CBP from the viewpoint of its interoperability as a whole. In other words, it describes and illustrates the "interoperability view" of CBP models.

The CBPipM is a complementary modelling approach for the ATHENA CBP modelling. Its premises are:

- $\quad$ The CBPipM is not a new modelling notation, but a modelling concept, which may be realized by extending (through profiling) the state-of-the-art modelling notations, e.g. BPMN, SoaML.

- $\quad$ The CBPipM models use the business logic and semantics already captured in CBP models but do not redefine them.

- The CBPipM is applicable in a semantically integrated, unified, or federated environment.

- $\quad$ The CBPipM focuses solely on business process interoperability modelling in a strong relationship to CBP models.

- The CBPipM model elements are definable by using CBP meta-model elements.

- The CBPipM suppresses business process centric model information (visualisation).

- $\quad$ The CBPipM supports vertical top-down (CIM -> PIM -> PSM -> IM) and bottom-up model transformations.

The CBPipM introduces the following modelling elements:

- $\quad$ state object

- A model stereotype for the process data.

- Trans-operation and trans-mapping 
- Model stereotypes for the interaction incorporating the transactions, particularly between View Process / Private Process pairs. Trans-operations are actions between COIN workflows and workflows of partner organization. Trans-mappings are co-local actions, i.e. they are not crossorganizational transactions. They are to be used particularly for semantic compatibility through invoking COIN semantic mediation services.

Using the state objects, trans-operations, and trans-mappings any cross-organization business interoperability workflow can be illustrated completely.

Figure 3 illustrate the relationship between a common CBP model and a CBPipM model. Note that, a fully automated M2M transformation from CBP model to CBPipM should be possible in the modelling environment.

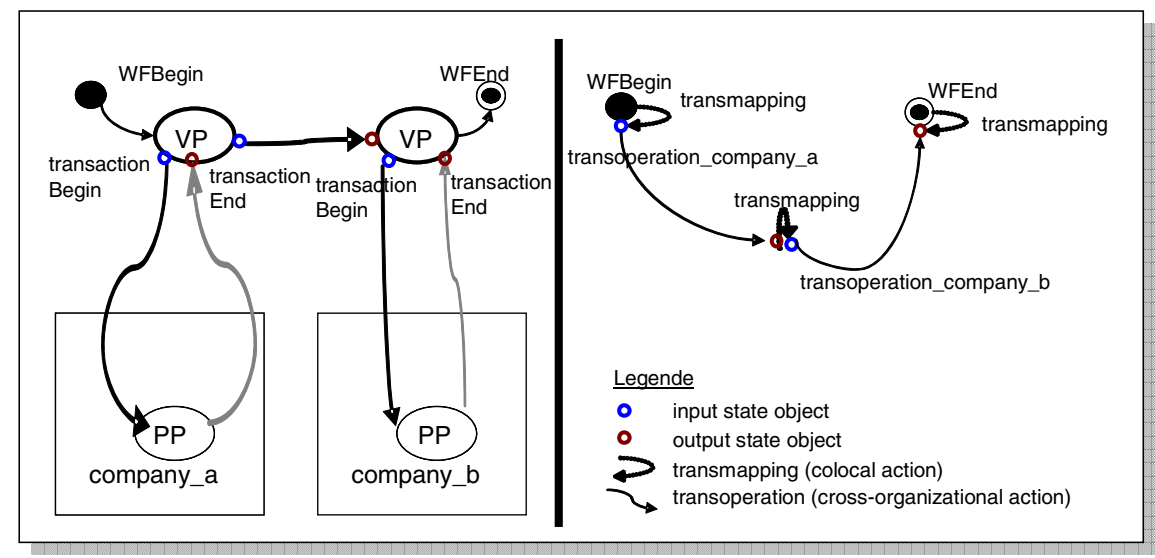

Fig. 3. CBP model vs. CBPip model

\section{Towards EI Service Utilities}

The COIN software model is built on software-as-a-service (SaaS) and software-as-aservice-utility (SaaS-U) concepts, which are emerging concepts for current and future networked enterprises.

SaaS is a model of software deployment where an application is hosted as a service provided to customers across the Internet. By eliminating the need to install and run the application on the customer's own computer, SaaS alleviates the customer's burden of software maintenance, ongoing operation, and support. Conversely, customers relinquish control over software versions or changing requirements.

Using SaaS also can conceivably reduce the up-front expense of software purchases, through less costly, on-demand pricing. From the software vendor's standpoint, SaaS has the attraction of providing stronger protection of its intellectual property and establishing an ongoing revenue stream. The SaaS software vendor may host the application on its own web server, or this function may be handled by a third-party application service provider (ASP). This way, end users may also reduce their investment on server hardware. 
SaaS is generally associated with business software and is typically thought of as a low-cost way for businesses to obtain the same benefits of commercially licensed, internally operated software without the associated complexity and high initial cost. Many types of software are well suited to the SaaS model, where customers may have little interest or capability in software deployment, but do have substantial computing needs. Application areas such as Customer Relationship Management, video conferencing, human resources, IT service management, accounting, IT security, web analytics, web content management and e-mail are some of the initial markets showing SaaS success. The distinction between SaaS and earlier applications delivered over the Internet is that SaaS solutions were developed specifically to leverage web technologies such as the browser, thereby making them web-native. The data design and architecture of SaaS applications are specifically built with a 'multi-tenant' backend, thus enabling multiple customers or users to access a shared data model. This further differentiates SaaS from client/server or ASP solutions because SaaS providers are leveraging enormous economies of scale in the deployment, management, support and through the Software Development Lifecycle.

The ongoing European research is trying to make a new implementation of the SaaS vision, a step forward in a new field of interoperability among collaborative enterprises, supporting the various collaborative business forms, from supply chains to business ecosystems, and becoming for them like a utility, a commodity, the socalled Interoperability Service Utility (ISU).

The ISU challenge is addressed by COIN by providing a service infrastructure for Enterprise Interoperability in the business context of Enterprise Collaboration. This will not just create a service platform, but mainly a new business concept - the Software-as-a-Service Utility (SaaS-U) model.

The SaaS-U paradigm fits well with the ISU concepts and can be seen as a software application delivery model where a software vendor develops Web-native software services and hosts and operates them for use by its customers over the Internet. Customers do not pay for owning the software itself any longer but rather for using it on-demand. They use it through an API accessible over the Web and often written using Web services.

Furthermore, the SaaS-U paradigm also fits well with modern service-oriented architecture (SOA) that aim to promote software development in a way that leverages the construction of dynamic software systems and which can easily adapt to volatile user environments and be easily maintained as well. SOA enables flexible connectivity of applications by representing every application as a service with a standardized interface. This enables them to exchange structured information quickly and flexibly. This flexibility enables new and existing applications to be easily and quickly combined to address changing business needs, and the ability to easily combine and choreograph applications allows IT services to more readily reflect business processes.

In order to transform a generic (web) service into a utility it is mandatory to describe and understand what are the requirements that "make" a utility. The COIN consortium since now has identified the following requirements:

- General Applicability. To satisfy a generic, common need

- Standards Based. To adopt known and recognized international or domain standards. 
- Critical Mass of Providers. To attract a certain number of service providers which could be interested in providing such a service.

- Ubiquitous Access. To be universally accessible.

- Guaranteed SLAs (Service Level Agreement). To be simultaneously accessible by anybody under guaranteed service levels.

- Simple Configuration. To require minimum configurations to be put in to action.

- Simple Outcomes. To be simply accessed by all and to provide clear and simple outcomes.

- Simple Verification \& Validation. To allow easily testing, verification and simulation of the declared functionality.

- Low cost. To be available at low cost, under subscription or pay-per-use models.

- Abundantly available. To be available generally to all, with no rivalry.

- Public Good. To be a public good, not in the exclusive hands of single private entity.

\section{Ongoing Activities and Preliminary Results}

Interoperability between processes also implies the capability of the two processes to exchange messages. The two processes manage incoming and outgoing messages in their own formats, which can be incompatible, for instance, in terms of terminology, and data structuring. Harmonization of messages between the data organization of the sender and the receiver is needed. To this end, a semantics-based approach for business documents reconciliation (this task is part of the COIN sub-project "Information Interoperability services (IIS)") will support the semantic process interoperability. The semantic reconciliation assumes the existence (or the creation) of a shared ontology, among application that intend to communicate, as a common reference. The schema of the documents to be exchanged are mapped against the ontology (semantic annotation) in order to build transformation rules that allow the transformation of a document expressed in terms of the specific application format, to and from the ontology representation. Such rules are then applied when instances of document are actually exchanged. The activity of IIS starts from the semantic reconciliation suite developed in the ATHENA [3] and aim at enhancing it in order to provide an automatic support to the semantic mapping.

\subsection{Tooling: Research, Candidates and Evaluation in Respect to SaaS}

The CBPip process is a complex task that starts with a modelling activity and finish with an execution activity. Currently the COIN consortium is concentrating in the modelling phase. The choice of the tools to be used during this phase is critical if we want to enhance the SaaS and SaaS-U models.

The evaluation of the tools to be used in the modelling phase has to respect the following requirements:

- Open source: the chosen tools must be completely free and usable.

- Standard based: the tools must work with standard formats recognised by international bodies. 
- Completely web-based: the users must not be required to install anything. Everything must be accessible from everywhere with a simple browser.

There are few candidates that respect all of the requirements specified above.

For the modelling tool the COIN consortium is evaluating the following tools:

- bxModeller [7]

- $\quad$ Oryx [8]

- ProcessMaker [9]

All the above mentioned tools are BPMN designers, which is the modeling language chosen for the CBPip process.

\subsection{Transformation from Private Process to View Process}

The aim of a transformation of a private process into a view process is to ease the specification of a public process which other companies can use and compose in a bigger setting; this public process will provide enough information and operations in order to call the private process, which will perform the real action.

The current version of the transformation, in MOFScript [10], implements a nearly complete privacy of the private process, meaning only the necessary operations remain during the transformation. COIN will study other means to lower such privacy, allowing for instance a partial view of the sub-processes that form the private process.

Figure 4 illustrates the transformation into a view process. This transformation works as follow:

- Copy each public operations from the View Process into the Public Process;

- Provide an operation in the View Process, which will perform the call to the Private Process

- $\quad$ Provide an operation in the View Process, which will be informed of the end of the Private Process

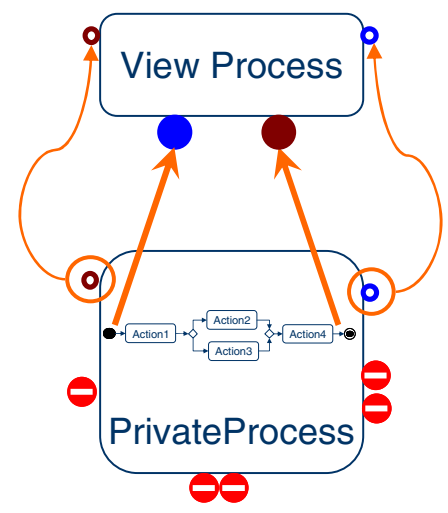

- input state object

- output state object

process start

process end

private operation

$\rightarrow$ transformation

Fig. 4. Transformation of a private process into a view process 


\subsection{CBPip Post-execution Analysis with CBPip Modeling}

With the means of CBPipM described in the previous section, the event log files of the IBM WebSphere Process Server [14] are roughly analyzed as a first step to the proof-of-concept. This section describes the preliminary identified pros and contras of CBPipM approach, where the lists are by no means complete (and proven) yet.

\section{PRO arguments:}

- "interoperability" as a model element associated with a formal specification and visualization which can be utilized for "post business process execution analysis (evidence search for a successfully executed CBP PSM)"

- The CBPip Framework described in the previous section can be extended in order to support the concepts of CBPipM

- CBPipM enables business process modeling and analysis on PSM and PIM levels, e.g. BPEL

- A new modeling technique to research on.

- Concentrates on basic interoperability issues: data, data semantic mapping and peek-to-peek transactions

- $\quad$ Provides model element unification, therefore applicability to both heterogeneous and homogeneous interoperability model transformation.

- Exposes multiple points to integrate appropriate semantic annotation and semantic mediation interoperability, human collaboration services.

- $\quad$ Aligned to UML2 modeling concepts, e.g. object flows.

\section{CONTRA arguments:}

- $\quad$ additional feasibility effort (proof of concept)

- CBPipM's consensus to business interoperability service architecture

- $\quad$ additional meta-modeling and model transformation effort

\section{Conclusions and Future Work}

The future work will concentrate on the three work packages, namely " BP modeling enhancement", "Transformation from Private Process to View Process", and "CBPip post-execution analysis", that are described in the following subordinated sections.

Furthermore, there are two topics that will gain momentum in the future: applying semantic annotation and usage of COIN semantic services, and integration of the deliverables of the work packages "Transformation into a View Process" and "CBPip post-execution analysis" as "BP modeling enhancements".

\subsection{BP Modeling Enhancement: Next Steps}

The COIN choice for the modelling tool is bxModeller [7] because among the different candidates proposed it is the one with the clearest architecture and uses the most known technologies.

The first step for the modelling enhancements is the customization of such modeller to allow the users to define private and public attributes on the activities and perform the call to the PP/VP transformation services. 


\subsection{Transformation into a View Process: Next Steps}

The current version of the transformation allows only a high level of privacy of the processes, as it focuses only on public / private operations, providing a 'black box view' of the private process. We are currently investigating in ways to produce a more 'grey box view', where the View Process will show a partial control flow of the private process. This implies transformation rules for operations to monitor the state of the private process.

\subsection{CBPip Post-execution Analysis: Next Steps}

Regarding to CBPip post-execution analysis with CBPip Modeling, the work will continue on to specify the CBPipM concepts precisely and in detail, to define the CBPipM meta-model with a given modeling notation, e.g. BPMN, SoaML, and to define and describe transformation formalisms text-2-model, e.g. event log structure to UML transformation. Additionally, the verification of CBP execution by the means of CBPipM will be investigated.

\section{References}

1. COIN, COIN Home Page, COIN IP, http : / /www. coin-ip.eu/ (last visited 2009)

2. ECOLEAD, ECOLEAD Home Page, http: / / ecolead.vtt. fi / (last visited 2009)

3. ATHENA, ATHENA Home Page, ATHENA IP, http://www.athena-ip.org/ (last visited 2007)

4. SHAPE, SHAPE Home Page, http : / /www . shape-project. eu / (last visited 2009)

5. MDA, Model Driven Architecture, Object Management Group (OMG), http : / / www . omg . org / mda / (last visited 2009)

6. BPMN, Business Process Modeling Notation, http://www.omg.org/bpmn/ (last visited 2009)

7. bxModeller, an open source web-based application tool for business process modeling, http://semantics.eng.it/bxmodeller/http: //semantics.eng.it/ bxmodeller/ (last visited 2009)

8. Oryx, an open source web-based application tool for business process modeling, http: / / bpt . hpi . uni-potsdam. de/Oryx/BPMN (last visited 2009)

9. ProcessMaker, an open source web-based application tool for business process management and workflow modeling, http: / / www . processmaker. com/ (last visited 2009)

10. Oldevik, J., Neple, T., Grønmo, R., Aagedal, J., Berre, A.: Toward Standardised Model to Text Transformations. In: European Conference on Model Driven Architecture - Foundations and Applications (ECMDA), Nuremberg (November 2005)

11. SoaML, Service oriented architecture Modeling Language, http: / / www . omg .org/docs / ad/08-08-04.pdf (last visited 2009)

12. BPEL, Web Services Business Process Execution Language, http: / / www . oasis-open.org/specs / \#wsbpelv2 . 0 (last visited 2009)

13. Elvesæter, B., Del Grosso, E., Capellini, A., Taglino, F., Benguria, G.: Towards Enterprise Interoperability Service Utilities. In: IWEI 2008 workshop (2008)

14. IBM WebSphere, a business process execution engine, http: / /www-01.ibm.com/software/integration/wps / (last visited 2009) 